\title{
Electronic supplementary
}

\section{For}

\section{A Macroporous 3D Scaffold with Self-Fitting Capability for Effectively Repairing Massive Rotator Cuff Tear}

Liren Wang ${ }^{\mathrm{a}}$, Yuhao Kang ${ }^{\mathrm{a}}$, Sihao Chen ${ }^{\mathrm{b}}$, Xiumei Moc, Jia Jianga, Xiaoyu Yan, Tonghe Zhu**,a, Jinzhong Zhao,,a

Email: jzzhao@sjtu.edu.cn (J. Zhao), zhutonghe@sjtu.edu.cn (T. Zhu)

* Corresponding author

Number of pages: 2

Nmber of figures: 0

\section{Materials}

Polycaprolactone (PCL, $\left.\mathrm{M}_{\mathrm{w}}=80,000\right)$ and polycaprolactone diol (HO-PCL-OH, $\left.\mathrm{M}_{\mathrm{w}}=2,000\right)$ were purchased from Sigma-Aldrich Trading Co., Ltd. (Shanghai, China). PEUU was synthesized from hexamethylene dissocyanate (HDI), and polycaprolactone diol (HO-PCL-OH) with 1', 4'butanediamine chain extension as done in our previous research. Alpha- Minimum Essential Medium ( $\alpha$-MEM), phosphate buffer saline (PBS, $\mathrm{pH}=7.4$ ), anhydrous dimethylsulfoxide (DMSO), 4', 6'-diamidino-2'-phenylindole (DAPI) were purchased from Sigma-Aldrich Trading Co., Ltd. (Shanghai, China). Penicillin-streptomycin, trypsin was purchased from Keygene BioTECH (China). Cell Counting Kit-8 (CCK-8) were purchased from Dojindo (Japan).

\section{Characterization of the PEUU and PCL scaffold}

Fourier transform infrared (FTIR) spectra was conducted with a Nicolet 6700 spectrometer (Thermo, USA) in a wavenumber range of $400-4000 \mathrm{~cm}^{-1}$. The surface and cross-sectional morphology of the prepared scaffolds was observed by scanning electron microscopy (SEM) (Phenom XL, Netherlands). Cross-sections of scaffolds were gained through cutting the samples after immersing in liquid nitrogen. Image J software (National Institutes of Health, USA) was used to calculate the pore sizes from cross-sectional SEM images. The water contact angle (WCA) of each sample was measured three times using a contact angle instrument (OCA 40, Dataphysics, Germany) when the 
droplet was stable at $23 \pm 0.5{ }^{\circ} \mathrm{C}$ and at a relative humidity of $30 \% \pm 2 \%$. Thermal properties were measured by using a STAPT-1000 instrument (Linseis, German). Samples (3-5 mg) were heated from $23{ }^{\circ} \mathrm{C}$ to $500{ }^{\circ} \mathrm{C}$ at a heating rate of $10{ }^{\circ} \mathrm{C} / \mathrm{min}$ under a flow of nitrogen $(40 \mathrm{~mL} / \mathrm{min})$. The mechanical properties of scaffolds were tested by a material testing machine (H5K-S, Hounsfield, UK) with a stretching speed of $1 \mathrm{~mm} / \mathrm{min}$.

\section{Micro-CT analysis}

The newly formed subchondral bones on the greater tubercles were evaluated using microcomputed tomography (eXplore Locus SP; GE Healthcare, London, ON, Canada). Each specimen was scanned at a voltage of $270 \mathrm{~mA}, 90 \mathrm{kV}$ and a $18 \mu \mathrm{m}$ voxel size. CT vox software (Skyscan) was used for three-dimensional reconstruction. The region of interest (ROI) was selected as the footprint of rotator cuff. Bone volume fraction (BV/TV, \%), trabecular number $\left(\mathrm{Tb} . \mathrm{N}, \mathrm{mm}^{-1}\right)$ and trabecular separation (Tb.Sp, mm)of each specimen were analyzed by CT-Analyser Software (Skyscan).

\section{Histological and histochemical analysis}

After fixation in $10 \%$ formalin for 7 days, complexes from experiment group and sham operation group ( $\mathrm{n}=3$ for each group) was decalcified, dehydrated, and embedded. Five-micrometer serial sections were cut for H\&E, Masson, Safranin O/Fast Green and histochemical staining of Col I evaluation. The images of the H\&E, masson and Safranin O/Fast Green, and histochemical staining of Col I staining sections were taken by a microscope (DM4000B; Leica Microsystems). In accordance with our previous studies, semiquantitative analysis of tendon maturing system, collagen fiber alignment, and collagen transformation was conducted. Metachromasia analysis was used as an indicator of mature fibrocartilage. The total area of new fibrocartilage formation at the insertion site was determined by outlining the area of metachromasia at a total magnification of 100 . The total area of metachromasia for each specimen was measured using the Image $\mathrm{J}$ imaging software program (National Institutes of Health, Bethesda, MD, USA) and then recorded as squared micron.

\section{Biomechanical testing}

Complexes from experiment group and sham operation group ( $\mathrm{n}=3$ for each group) for biomechanical testing were stored at $-80{ }^{\circ} \mathrm{C}$ freezer and thawed at $4{ }^{\circ} \mathrm{C}$ over night before biomechanical testing. The distal end of humerus of each complex was fixed by two $\varphi 2.00 \mathrm{~mm}$ 
Kirschner wires and the proximal end of regenerated tendon was fixed by a clamp. Traction force was applied along the regenerated tendon tissue. After suture were split, 10 consecutive cycles were conducted in order to define a standard "zero load" as previously described. After preconditioning, the ultimate load to failure of the scapula-rotator cuff tendon-humerus was recorded at a stretching rate of $1.00 \mathrm{~mm} / \mathrm{min}$. The load and displacement curve data were automatically recorded by the machine data system. The stiffness and ultimate stress were then calculated. 